\title{
THE ULTRAFILTER CHARACTERIZATION OF HUGE CARDINALS
}

\author{
ROBERT J. MIGNONE'
}

\begin{abstract}
A huge cardinal can be characterized using ultrafilters. After an argument is made for a particular ultrafilter characterization, it is used to prove the existence of a measurable cardinal above the huge cardinal, and an ultrafilter over the set of all subsets of this measurable cardinal of size smaller than the huge cardinal. Finally, this last ultrafilter is disassembled intact by a process which often produces a different ultrafilter from the one started out with. An important point of this paper is given the existence of the particular ultrafilter characterization of a huge cardinal mentioned above these results are proved in Zermelo-Fraenkel set theory without the axiom of choice.
\end{abstract}

Introduction. The notion of a huge cardinal was first introduced by Kunen. Its primary definition is given in terms of elementary embeddings. And just as measurable and supercompact cardinals have ultrafilter characterizations in ZFC, a huge cardinal can also be characterized by ultrafilters.

In $\S 1$, after the elementary embedding definition for a huge cardinal and two equivalent ultrafilter characterizations are given, an argument is made for one characterization above the other in instances where the axiom of choice is not available. Once an ultrafilter characterization for $\kappa$ being huge is settled upon-which defines $\kappa$ as huge if for some $\lambda>\kappa$ there exists a $\kappa$-complete, normal ultrafilter over $\{x \subset \lambda: \bar{x}=\kappa\}$-two theorems are proved in $\$ 2$ which state that providing such an ultrafilter exists over $\{x \subset \lambda: \bar{x}=\kappa\}$, then $\lambda$ is measurable and $\kappa$ is $\lambda$-supercompact. These are both well-known theorems in ZFC, but here they are established in ZF without the axiom of choice.

Finally, given an ultrafilter over $P_{\kappa} \lambda$ it can be restricted to ultrafilters over $P_{\kappa} \alpha$ for $\kappa<\alpha<\lambda$. These ultrafilters in turn can be "glued together" with a measure on $\lambda$ to again produce an ultrafilter over $P_{\kappa} \lambda$. In general, the ultrafilter over $P_{\kappa} \lambda$ obtained by this method is not the same one which is started out with (see [2]). In $\S 3$ it is shown that if the ultrafilter constructed over $P_{\kappa} \lambda$ in $\$ 2$ is restricted to ultrafilters over $P_{\kappa} \alpha$ for $\kappa<\alpha<\lambda$, then glued together with the measure constructed on $\lambda$ in this same section, the glued together ultrafilter is the same as the one started out with.

Received by the editors March 9, 1983 and, in revised form, May 9, 1983. Some of the results from this paper were presented by the author at the following meetings: AMS annual meeting, January 1983, Denver, Colorado, and Summer meeting of the ASL, August 1982, Florence, Italy.

1980 Mathematics Subject Classification. Primary 03E55.

${ }^{1}$ Research was partially supported by a College of Charleston Foundation grant. The author wishes to express his appreciation to The Institute for Advanced Study for their invitation and hospitality during the summer of 1982 , where a portion of this research was completed. 
$\S \S 2$ and 3 answer some questions which arose at Cornell University in the summer of 1982 during the American Mathematical Society Research Institute.

For the most part the notation used here is consistent with [3]. However, uses of two types of notation may be contrary to customary practice and so need special note. For $\kappa$ an uncountable cardinal and $\kappa<\lambda,[\lambda]^{\kappa}=\{x \subset \lambda:|x|=\kappa\}$ and $(\lambda)^{\kappa}=\{x \subset \lambda: \bar{x}=\kappa\}$, where $|x|$ is the cardinality of $x$ and $\bar{x}$ is the order-type of $x$. As usual, $P_{\kappa} \lambda=\{x \subset \lambda:|x|<\kappa\}$. Also, consistent with [7], a normal ultrafilter over $P_{\kappa} \lambda,[\lambda]^{\kappa}$ or $(\lambda)^{\kappa}$ includes the property of being fine. Given $x \in(\lambda)^{\kappa}$ and $\eta<\kappa$, $x(\eta)$ will denote the $\eta$ th element of $x$ in the ascending enumeration of $x$, and $\eta$ will be called the index of $x(\eta)$.

For definitions of the basic properties of set theory and large cardinals the reader is referred to [3]. For background specific to this paper the reader is referred to [7].

1. Let $\kappa$ be an uncountable cardinal. Then $\kappa$ is said to be huge if there is an elementary embedding $j: V \rightarrow M$ such that $\kappa$ is the critical point of $j$ (this means $\kappa$ is the first ordinal moved by $j$ ) and ${ }^{j(\kappa)} M \subset M$.

In $\mathrm{ZFC}, \kappa$ being huge is equivalent to the following two ultrafilter characterizations.

Characterization 1 . There exists a $\lambda>\kappa$ and a normal, $\kappa$-complete ultrafilter over $[\lambda]^{\kappa}($ see $[3])$.

Characterization 2 . There exists a $\lambda>\kappa$ and a normal, $\kappa$-complete ultrafilter over $(\lambda)^{\kappa}$ (see [7]).

In the case of supercompact cardinals when choice is absent, typically the ultrafilter characterization is used. The same thing can be done for the notion of a huge cardinal. But in the case of a huge cardinal there are two characterizations which are equivalent in ZFC to the elementary embedding definition. However, on further investigation, in the absence of the axiom of choice, one of these surfaces as the more desirable characterization.

Let $U$ be a normal, $\kappa$-complete ultrafilter over $(\lambda)^{\kappa}$. Then $U$ generates a normal, $\kappa$-complete ultrafilter over $[\lambda]^{\kappa}$, regardless of choice. And with choice if $V$ is a normal, $\kappa$-complete ultrafilter over $[\lambda]^{\kappa}$, and $V^{\prime}$ is defined over $[\lambda]^{\kappa}$ by $A \in V^{\prime}$ if and only if $j_{V}^{\prime \prime} \lambda \in j(A)$, then $V=V^{\prime}$. Using this in a straightforward manner it can be shown that for any normal, $\kappa$-complete ultrafilter $V$ over $[\lambda]^{\kappa},\left\{x \in[\lambda]^{\kappa}: \bar{x}=\kappa\right\} \in V$. So $(\lambda)^{\kappa} \in V$. This contrasts with what follows.

In Zermelo-Fraenkel set theory with the axiom of determinateness (ZF + AD), by results of Solovay and Martin $\boldsymbol{N}_{2}$ is measurable (see [2]). Let $\mu$ be a normal measure over $\boldsymbol{\kappa}_{2}$. Define a normal, $\boldsymbol{\kappa}$-complete ultrafilter $U$ over $\left[\boldsymbol{\kappa}_{2}\right]^{\boldsymbol{\kappa}_{1}}$ as follows: for $A \subset\left[\boldsymbol{\aleph}_{2}\right]^{\boldsymbol{\kappa}_{1}}, A \in U$ if and only if $A \cap \boldsymbol{\aleph}_{2} \in \mu$. Clearly, $\left\{x \in\left[\boldsymbol{\aleph}_{2}\right]^{\boldsymbol{\kappa}_{1}}: \bar{x}=\boldsymbol{\aleph}_{1}\right\} \notin U$.

Summarizing, in ZF a normal, $\kappa$-complete ultrafilter over $(\lambda)^{\kappa}$ is essentially a normal, $\kappa$-complete ultrafilter over $[\lambda]^{\kappa}$. But in ZF + AD there exists $\lambda, \kappa$ and a normal, $\kappa$-complete ultrafilter $V$ over $[\lambda]^{\kappa}$ such that $(\lambda)^{\kappa} \notin V$. This stands as an intrinsic argument for using Characterization 2 as the appropriate ultrafilter characterization of a huge cardinal. In this sense the question of the "hugeness" of $\aleph_{1}$ in $\mathrm{ZF}+\mathrm{AD}$ is still unresolved. 
2. If $\kappa$ is huge and $j: V \rightarrow M$ such that $\kappa$ is the critical point and ${ }^{j(\kappa)} M \subset M$, then it is clearly the case that $\kappa$ is $\alpha$-supercompact for $\kappa<\alpha<j(\kappa)$. Recent results by Barbanel, DiPrisco and Tan extend this to include that $\kappa$ is $j(\kappa)$-supercompact. Their method of proof requires the axiom of choice and is established in the following way: If $\kappa$ is huge and $j: V \rightarrow M$ is a witness to this, then $j(\kappa)$ is measurable in $V$ (see [1]). Using the fact that $\kappa$ is $\alpha$-supercompact for $\kappa<\alpha<j(\kappa)$, ultrafilters on $P_{\kappa} \alpha$ for $\kappa<\alpha<j(\kappa)$ can be "glued-together" via a normal measure on $j(\kappa)$, yielding a normal, $\kappa$-complete ultrafilter over $P_{\kappa} j(\kappa)$ (see [2 or 6]).

The following theorem achieves the same conclusion from the ultrafilter characterization of huge without the axiom of choice. (Note. In ZFC the elementary embedding $j: V \rightarrow M$ is used to define an ultrafilter over $(\lambda)^{\kappa}$ where $j(\kappa)=\lambda$. Hence without the axiom of choice $\lambda$ replaces $j(\kappa)$.)

THEOREM 1. (ZF) Let $\kappa$ be a cardinal and $\lambda>\kappa$. If there exists a normal, $\kappa$-complete ultrafilter $\hat{U}$ over $(\lambda)^{\kappa}$, then $\kappa$ is $\lambda$-supercompact.

Proof. Using the methods of [2] for constructing a $\kappa$-complete, normal ultrafilter over $\kappa$ from a $\kappa$-complete, normal ultrafilter over $P_{\kappa} \lambda$, the same construction can be obtained starting with a normal, $\kappa$-complete ultrafilter over $(\lambda)^{\kappa}$. So assume $\nu$ is a $\kappa$-complete, normal ultrafilter over $\kappa$. Define $U$ over $P_{\kappa} \lambda$ as follows: for $A \subset P_{\kappa} \lambda$,

$$
A \in U \text { if and only if }\left\{x \in(\lambda)^{\kappa}:\{\eta: x \cap x(\eta) \in A\} \in \nu\right\} \in \hat{U} .
$$

The verification that $U$ is a $\kappa$-complete, fine ultrafilter is routine. The only property that will be dealt with here is normality. Let $f: P_{\kappa} \lambda \rightarrow \lambda$ be such that $\left\{y \in P_{\kappa} \lambda\right.$ : $f(y) \in y\} \in U$. Then for $x \in(\lambda)^{\kappa}$, let $g(x)$ be the unique $\alpha$ such that $\{\eta$ : $f(x \cap x(\eta))=\alpha\} \in \nu$, which is guaranteed by the normality of $\nu$. Now, by the normality of $\hat{U}$, there is an $\alpha<\lambda$ such that $g^{-1}(\alpha) \in U$ so $f^{-1}(\alpha) \in U$.

Unlike the glued-together ultrafilter over $P_{\kappa} \lambda$, the ultrafilter over $P_{\kappa} \lambda$, constructed in the proof of Theorem 1 as a restriction of the ultrafilter over $(\lambda)^{\kappa}$, did not require that $\lambda$ be measurable for its existence. However, just as $\kappa$ being huge yields $j(\kappa)$ measurable in ZFC, $\kappa$ being huge in the sense of Characterization 2 yields $\lambda$ measurable in $\mathrm{ZF}$ without the axiom of choice.

THEOREM 2. (ZF) Let $\kappa$ be a cardinal and $\lambda>\kappa$. If there exists a normal, $\kappa$-complete ultrafilter $\hat{U}$ over $(\lambda)^{\kappa}$, then there is a $\lambda$-complete, normal ultrafilter over $\lambda$. Hence $\lambda$ is measurable.

Proof. Again assume $\kappa$ is measurable. Let $\nu$ be a $\kappa$-complete, normal ultrafilter over $\kappa$. Define $\mu$ over $\lambda$ as follows: for $a \subset \lambda$,

$$
a \in \mu \quad \text { if and only if } \quad\left\{x \in(\lambda)^{\kappa}:\{\eta: x(\eta) \in a\} \in \nu\right\} \in \hat{U} .
$$

This defines a $\lambda$-complete, normal ultrafilter over $\lambda$. The verification that $\mu$ is an ultrafilter is routine. For normality let $f: \lambda \rightarrow \lambda$. Assume $a=\{\alpha<\lambda: f(\alpha)<\alpha\} \in \mu$. Set

So $\hat{A} \in \hat{U}$.

$$
\hat{A}=\left\{x \in(\lambda)^{\kappa}:\{\eta: x(\eta) \in a\} \in \nu\right\} .
$$


Claim. $\{x \in \hat{A}: f(x(\eta)) \in x$ for every $\eta<\kappa\} \in \hat{U}$.

Proof of Claim. Set

$$
\hat{A}_{\alpha}=\{x \in \hat{A}: f(\alpha) \in x\} \quad \text { for every } \alpha<\lambda .
$$

Then $\hat{A}_{\alpha} \in \hat{U}$. By normality

$$
\Delta_{\alpha<\lambda} \hat{A}_{\alpha}=\{x \in \hat{A}: \alpha \in x \text { implies } f(\alpha) \in x\} \in \hat{U} .
$$

Since $x(\eta) \in x$ for every $\eta<\kappa$ the claim is proved.

Set

$$
\hat{E}=\{x \in \hat{A}: f(x(\eta)) \in x \text { for every } \eta<\kappa\} .
$$

For $x \in \hat{E}$, set

$$
b_{x}=\{\eta: x(\eta) \in a\} \cap\{\eta: f(x(\eta))<x(\eta)\} .
$$

So $b_{x} \in \nu$. Next, define $g_{x}: \kappa \rightarrow \kappa$ by

$$
g_{x}(\eta)= \begin{cases}\text { index of } f(x(\eta)) \text { in } x & \text { if } \eta \in b_{x}, \\ \varnothing & \text { otherwise. }\end{cases}
$$

Hence $x\left(g_{x}(\eta)\right)=f(x(\eta))<x(\eta)$ for all $\eta \in b_{x}$. By the normality of $\nu$ there exists a $\gamma_{x}<\kappa$ and $c_{x} \in \nu$ such that $c_{x} \subset b_{x}$ and $g_{x}(\eta)=\gamma_{x}$ for $\eta \in c_{x}$. Define $F:(\lambda)^{\kappa} \rightarrow \lambda$ by

$$
F(x)=\left\{\begin{array}{l}
x\left(\gamma_{x}\right) \text { for } x \in \hat{E}, \\
\varnothing \quad \text { otherwise }
\end{array}\right.
$$

So $F(x) \in x$ for each $x \in \hat{E}$. By the normality of $\hat{U}$ there exists an $\hat{H} \in \hat{U}$ and $\xi<\lambda$ such that $\hat{H} \subset \hat{E}$ and $F(x)=\xi$ for each $x \in \hat{H}$. Finally set

$$
c=\left\{x(\eta): x \in \hat{H} \text { and } \eta \in c_{x}\right\} .
$$

Hence $c \in \mu$ and for each $\alpha \in c, \alpha=x(\eta)$ for some $x \in \hat{H}$ and $\eta \in c_{x}$. Therefore

$$
f(\alpha)=f(x(\eta))=x\left(g_{x}(\eta)\right)=x\left(\gamma_{x}\right)=F(x)=\xi .
$$

Since $\mu$ is normal, a standard argument can now be applied to show that it is $\lambda$-complete.

This completes the proof of the theorem.

3. Theorem 1 shows that the existence of a $\kappa$-complete, normal ultrafilter over $(\lambda)^{\kappa}$ provides a $\kappa$-complete, normal ultrafilter over $P_{\kappa} \lambda$. If, in turn, this ultrafilter over $P_{\kappa} \lambda$ is used to define $\kappa$-complete, normal ultrafilters over $P_{\kappa} \alpha$ for $\kappa<\alpha<\lambda$, then these ultrafilters can be glued together with the measure on $\lambda$ provided by Theorem 2 to again give a $\kappa$-complete, normal ultrafilter over $P_{\kappa} \lambda$. The next theorem shows that this process starts and finishes with the same ultrafilter.

Fix a $\kappa$-complete, normal ultrafilter $\hat{U}$ over $(\lambda)^{\kappa}$, and a normal measure $\nu$ on $\kappa$. Define $U$ over $P_{\kappa} \lambda$ as in the proof of Theorem 1: for $A \subset P_{\kappa} \lambda$,

$$
A \in U \text { if and only if }\left\{x \in(\lambda)^{\kappa}:\{\eta: x \cap x(\eta) \in A\} \in \nu\right\} \in \hat{U} .
$$


For each $\alpha$ such that $\kappa<\alpha<\lambda$ define $U_{\alpha}$ over $P_{\kappa} \alpha$ as follows: for $A \subset P_{\kappa} \alpha$,

$$
A \in U_{\alpha} \quad \text { if and only if } \quad\left\{y \in P_{\kappa} \lambda: y \cap \alpha \in A\right\} \in U .
$$

This defines a $\kappa$-complete, normal ultrafilter over $P_{\kappa} \alpha$ (see [2]).

Let $\mu$ be the normal, $\lambda$-complete ultrafilter over $\lambda$ defined from $\hat{U}$ and $\nu$ as in the proof of Theorem 2. That is, for $a \subset \lambda$

$$
a \in \mu \quad \text { if and only if } \quad\left\{x \in(\lambda)^{\kappa}:\{\eta: x(\eta) \in a\} \in \nu\right\} \in \hat{U} .
$$

The glued-together ultrafilter $U^{\prime}$, over $P_{\kappa} \lambda$, of the $U_{\alpha}$ 's via $\mu$ is defined by

$$
A^{\prime} \in U^{\prime} \quad \text { if and only if } \quad\left\{\alpha<\lambda: A^{\prime} \cap P_{\kappa} \alpha \in U_{\alpha}\right\} \in \mu .
$$

THEOREM 3. (ZF) If $U$ and $U^{\prime}$ over $P_{\kappa} \lambda$ are defined as in the previous three paragraphs, then $U=U^{\prime}$.

Proof. Let $\hat{U}$ over $(\lambda)^{\kappa}, \nu$ over $\kappa, \mu$ over $\lambda, U$ over $P_{\kappa} \lambda, U_{\alpha}$ 's over $P_{\kappa} \alpha$ for $\kappa<\alpha<\lambda$ and $U^{\prime}$ over $P_{\kappa} \lambda$ be defined as they are defined in the previous three paragraphs.

Claim 1. If $b \in \mu$ and $\hat{A}_{\alpha} \in \hat{U}$ for each $\alpha \in b$ and $\Delta_{\alpha \in b} \hat{A}_{\alpha}$ is defined by $\Delta_{\alpha \in b} \hat{A}_{\alpha}=\left\{x \in(\lambda)^{\kappa}: \alpha \in b\right.$ and $\alpha \in x$ implies $\left.x \in \hat{A}_{\alpha}\right\}$, then $\Delta_{\alpha \in h} \hat{A}_{\alpha} \in \hat{U}$.

Proof of Claim 1. Assume the contrary. That is, $\Delta_{\alpha \in h} \hat{A} \notin \hat{U}$. Set $\hat{B}=\{x \in$ $\left.(\lambda)^{\kappa}:\{\eta: x(\eta) \in b\} \in \nu\right\}$. So $\hat{B} \in \hat{U}$, and by this assumption, $\hat{B}-\Delta_{\alpha \in h} \hat{A}_{\alpha} \in \hat{U}$.

Define $F:(\lambda)^{\kappa} \rightarrow \lambda$ as follows:

$$
F(x)=\left\{\begin{array}{l}
\text { least } \alpha \text { in } x \cap b \text { such that } x \notin \hat{A}_{\alpha} \quad \text { if } x \in \hat{B}-\Delta_{\alpha \in b} \hat{A}_{\alpha}, \\
\varnothing \quad \text { otherwise. }
\end{array}\right.
$$

So $F(x) \in x$ for each $x \in \hat{B}-\Delta_{\alpha \in b} \hat{A}_{\alpha}$. By the normality of $\hat{U}$ there exists a $\delta<\lambda$ and a $\hat{C} \subset \hat{B}-\Delta_{\alpha \in b} \hat{A}$ such that $\hat{C} \in \hat{U}$ and $F(x)=\delta$ for each $x \in \hat{C}$. Take $x \in \hat{A}_{\delta} \cap \hat{C}$. So $x \in \hat{A}_{\delta}$, and $x \in \hat{C}$, so $F(x)=\delta$, which means $x \notin A_{\delta}$. This contradiction proves Claim 1.

Given $A \in U$, set $\hat{A}=\left\{x \in(\lambda)^{\kappa}:\{\eta: x \cap x(\eta) \in A\} \in \nu\right\}$, and for $x \in \hat{A}$, $b_{x}=\{\eta: x \cap x(\eta) \in A\}$. So $\hat{A} \in \hat{U}$ and $b_{x} \in \nu$. Finally set $a=\{x(\eta): x \in \hat{A}$ and $\left.\eta \in b_{x}\right\}$. So $a \in \mu$.

Claim 2. For $\hat{A}, b_{x}$ and $a$ as defined above, $\{\alpha \in a:\{x \in \hat{A}: x(\eta)=\alpha$ for some $\left.\left.\eta \in b_{x}\right\} \in \hat{U}\right\} \in \mu$.

Proof of Claim 2. Assume otherwise. For each $\alpha \in a$ set $\hat{A}_{\alpha}=\{x \in \hat{A}: x(\eta) \neq \alpha$ for all $\left.\eta \in b_{x}\right\}$. Then set $b=\left\{\alpha \in a: \hat{A}_{\alpha} \in \hat{U}\right\}$. By the assumption, $b \in \mu$. The previous claim yields $\Delta_{\alpha \in b} \hat{A}_{\alpha} \in \hat{U}$. So $\Delta_{\alpha \in b} \hat{A}_{\alpha} \cap \hat{A} \in \hat{U}$. Set $c=\{x(\eta): x \in$ $\Delta_{\alpha \in b} \hat{A}_{\alpha} \cap \hat{A}$ and $\left.\eta \in b_{x}\right\}$. Hence $c \cap b \in \mu$. For $\alpha \in c \cap b$, since $\alpha \in c$ take $x \in \Delta_{\alpha \in b} \hat{A}_{\alpha} \cap \hat{A}$ such that $x(\eta)=\alpha$ for some $\eta \in b_{x}$. Now $\alpha \in b$ and $\alpha \in x$ so $x \in \hat{A}_{\alpha}$. But this means $x(\eta) \neq \alpha$ for all $\eta \in b_{x}$. This contradiction proves Claim 2.

Set $d=\left\{\alpha \in a:\left\{x \in \hat{A}: x(\eta)=\alpha\right.\right.$ for some $\left.\left.\eta \in b_{x}\right\} \in \hat{U}\right\}$. By Claim 2, $d \in \mu$. For any $\alpha \in d:\left\{x \in \hat{A}: x(\eta)=\alpha\right.$ for some $\left.\eta \in b_{x}\right\} \in \hat{U}$, which implies

$$
\begin{aligned}
\left\{y \in A: y=x \cap x\left(\eta^{\prime}\right) \text { for some } x \in \hat{A}, \eta^{\prime} \in b_{x}\right. \\
\left.\quad \text { and such that } x(\eta)=\alpha \text { for some } \eta \in b_{x}\right\} \in U,
\end{aligned}
$$


which implies

$$
\begin{aligned}
& \left\{y \in A: y=x \cap x\left(\eta^{\prime}\right) \text { for some } x \in \hat{A} \text { and } \eta^{\prime} \in b_{x}-\xi_{\alpha},\right. \\
& \text { where } \left.\xi_{\alpha} \in b_{x} \text { such that } x\left(\xi_{\alpha}\right)=\alpha\right\} \in U,
\end{aligned}
$$

which in turn implies

$$
\begin{aligned}
& \left\{y \in A: y \cap \alpha=x \cap x\left(\eta^{\prime}\right) \cap \alpha=x \cap x\left(\eta^{\prime}\right) \cap x(\eta)=x \cap x(\eta)\right. \\
& \left.\quad \in A \cap P_{\kappa} \alpha\left(\text { since } x \in \hat{A}, \eta \in b_{x} \text { and } \eta<\eta^{\prime} \text { for almost all } \eta^{\prime} \text { in } b_{x}\right)\right\} \in U .
\end{aligned}
$$

That is,

$$
\left\{y \in A: y \cap \alpha \in A \cap P_{\kappa} \alpha\right\} \in U \text {. }
$$

Hence $A \cap P_{\kappa} \alpha \in U_{\alpha}$ for each $\alpha \in d$. So $\left\{\alpha<\lambda: A \cap P_{\kappa} \alpha \in U_{\alpha}\right\} \in \mu$. Therefore $A \in U^{\prime}$. Since $U$ is an ultrafilter, $U=U^{\prime}$.

In the constructions of ultrafilters preceding the statement of Theorem 3, an ultrafilter $U$ over $P_{\kappa} \lambda$ was restricted to ultrafilters over $P_{\kappa} \alpha$ for $\kappa<\alpha<\lambda$, and then glued together to yield $U$ over $P_{\kappa} \lambda$ back again. In ZFC, if $\kappa$ is supercompact this, in general, cannot be done for any ultrafilter $U$ over $P_{\kappa} \lambda$. This is a consequence of the following discussion. Consider the following definition of Jech (see [4]). Define $\left[P_{\kappa} \lambda\right]^{2}=\left\{\{x, y\}: x, y \in P_{\kappa} \lambda\right.$ and $\left.x \neq y\right\}$. An unbounded subset $A$ of $P_{\kappa} \lambda$ is called homogeneous for a function $F:\left[P_{\kappa} \lambda\right]^{2} \rightarrow 2$ if there is a $k<2$ such that for all $x, y \in A$ with $x \subsetneq y$ or $y \subsetneq x$, then $F(\{x, y\})=k$. A $\kappa$-complete, normal ultrafilter $U$ over $P_{\kappa} \lambda$ is said to have the partition property if for every $F:\left[P_{\kappa} \lambda\right]^{2} \rightarrow 2, U$ has a homogeneous set for $F$ as a member. Menas showed that if $\kappa$ is supercompact and $\lambda \geqslant \kappa$, then there are $2^{2^{\lambda \kappa}} \kappa$-complete, normal ultrafilters over $P_{\kappa} \lambda$ with the partition property (see [5]).

On the other hand, Solovay showed that if $U$ is a $\kappa$-complete, normal ultrafilter over $P_{\kappa} \lambda$, and $U_{\alpha}$ 's over $P_{\kappa} \alpha$ for $\kappa<\alpha<\lambda$ are defined by $A \in U_{\alpha}$ if and only if $\left\{y \in P_{\kappa} \lambda: y \cap \alpha \in A\right\} \in U$, then the glued-together ultrafilter over $P_{\kappa} \lambda$ of the $U_{\alpha}$ 's does not have the partition property (see [2]).

\section{REFERENCES}

1. J. B. Barbanel, C. A. DiPrisco and I. B. Tan, Many-times huge and supercompact cardinals, J. Symbolic Logic 49 (1984), 177- 187.

2. C. A. DiPrisco and J. Henle, On the compactness of $\boldsymbol{\aleph}_{1}$ and $\boldsymbol{\aleph}_{2}$, J. Symbolic Logic 43 (1978), 394-401.

3. T. J. Jech, Set theory, Academic Press, New York, 1978.

4. Some combinatorial problems concerning uncountable cardinals, Ann. Math. Logic 5 (1973), $165-198$.

5. T. K. Menas, $A$ combinatorial property of $P_{\kappa} \lambda$, J. Symbolic Logic 41 (1976), 225-233.

6. R. J. Mignone, Ultrafilters resulting from the axiom of determinateness, Proc. London Math. Soc. (3) 43 (1981), 582-605.

7. R. Solovay, W. Reinhardt and A. Kanamori, Strong axioms of infinity and elementary embeddings, Ann. of Math. Logic 13 (1978), 73-116.

Department of Mathematics, College of Charleston, Charleston, South Carolina 29424 